\title{
Valores en educación universitaria: equidad de género en parejas de doble ingreso
}

Values in University Education: Gender Equality in Double Income Couples

Valores na educação universitária: igualdade de gênero em casais de dupla renda

Flor de María García Martínez Universidad Autónoma de Zacatecas, Unidad Académica de Psicología, México florecitagama@hotmail.com https://orcid.org/0000-0003-3869-0169

Víctor Hugo Bañuelos García Universidad Autónoma de Zacatecas, Unidad Académica de Contaduría y Administración, México

bag_70@hotmail.com https://orcid.org/0000-0003-0888-4157

Blanca Isabel Llamas Félix Universidad Autónoma de Zacatecas, Unidad Académica de Contaduría y Administración, México isabelllamas@ymail.com https://orcid.org/0000-0002-0782-8340 


\section{Revista Iberoamericana \\ de las Ciencias Sociales y Humanísticas}

ISSN: $2395-7972$

\section{Resumen}

La educación es un requerimiento clave para el siglo XXI. Dadas las exigencias del mundo contemporáneo, la concepción de valores se encuentra dirigida al desarrollo de la personalidad del estudiante universitario, proceso complejo y contradictorio en el que intervienen diversos factores. Así pues, el objetivo de la presente investigación consistió en identificar valores en la educación universitaria que reconocieran la equidad de género en parejas de doble ingreso que laboraron en un departamento gubernamental del estado de Zacatecas en el año 2019. La investigación se desarrolló en un entorno cuantitativo de tipo transaccional. El nivel de investigación es relacional: se encontraron relaciones y discrepancias entre las variables de formación de valores y equidad de género. En los resultados alcanzados, se encontró, mediante la prueba de ji al cuadrado, relación entre el género y las actividades del hogar. Sin embargo, un índice sumativo simple de $91.07 \%$ y 1.79 \% consideró lo contrario. Por tanto, se concluyó que, en las parejas de doble ingreso que laboraron en este departamento gubernamental, existieron valores como la libertad, la igualdad, el respeto, la comunicación y la negociación, la equidad de género, los cuales subyacen a los derechos humanos fundamentales como forma de resolución de conflictos.

Palabras clave: equidad de género, pareja doble ingreso, universidad, valores.

\section{Abstract}

Education is a key requirement for the $21^{\text {st }}$ century. Given the demands of the contemporary world, the conception of values is directed to the development of the personality of the university student, a complex and contradictory process in which various factors intervene. Thus, the objective of this research was to identify values in university education that recognized gender equity in dual-income couples who worked in a government department in the state of Zacatecas in 2019. The research was developed in a transactional quantitative environment. The research level is relational: relationships and discrepancies were found between the variables of value formation and gender equity. In the results achieved, a relationship between gender and household activities was found through the chi-square test. However, a simple summative index of $91.07 \%$ and $1.79 \%$ considered the opposite. Therefore, it was concluded that, in the dual-income couples who worked in this government department, there were values such as freedom, equality, 


\section{Revista Iberoamericana \\ de las Ciencias Sociales y Humanísticas}

ISSN: $2395-7972$

respect, communication and negotiation, gender equality, which underlie the fundamental human rights as a form of conflict resolution.

Keywords: gender equity, double income couple, university, values.

\section{Resumo}

A educação é um requisito fundamental para o século 21. Atendendo às exigências do mundo contemporâneo, a concepção de valores está direccionada para o desenvolvimento da personalidade do estudante universitário, um processo complexo e contraditório no qual intervêm vários factores. Assim, o objetivo desta pesquisa foi identificar valores na formação universitária que reconhecem a igualdade de gênero em casais de dupla renda que trabalhavam em um departamento governamental do estado de Zacatecas em 2019. A pesquisa foi desenvolvida em um ambiente do tipo transacional quantitativo. O nível da pesquisa é relacional: foram encontradas relações e discrepâncias entre as variáveis de formação de valor e equidade de gênero. Nos resultados alcançados, foi encontrada relação entre gênero e atividades domésticas por meio do teste do qui-quadrado. No entanto, um índice somativo simples de $91,07 \%$ e 1,79\% considerou o contrário. Portanto, concluiu-se que, nos casais de dupla renda que trabalhavam neste órgão governamental, existiam valores como liberdade, igualdade, respeito, comunicação e negociação, eqüidade de gênero, que fundamentam os direitos humanos fundamentais como forma de conflito. resolução.

Palavras-chave: igualdade de gênero, casal de dupla renda, universidade, valores.

Fecha Recepción: Junio 2020

Fecha Aceptación: Diciembre 2020

\section{Introducción}

La educación superior en México trata de alcanzar patrones internacionales. De hecho, en la última década, el debate sobre el futuro de la educación superior ha estado presente en todo el mundo. Entre los temas más abordados, destaca el de los sistemas educativos, la necesidad de revisarlos y actualizarlos para enfrentar las demandas del mundo globalizado, así como la de prestar atención a estas para realizar las reformas educativas necesarias. El sistema de educación superior supone un compromiso del Gobierno en su nivel federal, estatal, así como municipal, de los poderes legislativos y de la 


\section{Revista Iberoamericana de las Ciencias Sociales y Humanísticas}

ISSN: $2395-7972$

sociedad civil, con la educación superior. Trazar la visión y plantear opciones para el futuro requiere de creatividad y objetividad.

La propuesta de la Asociación Nacional de Universidades de Institución Superior (Anuies) para coadyuvar al desarrollo y consolidación del actual sistema de educación superior parte de que se requiere cambiar de manera elemental para dar respuesta a la equidad, eficiencia y calidad; es decir, alude al conjunto de demandas planteadas por la sociedad mexicana y las transformaciones del entorno nacional e internacional, donde las exigencias educativas son cada vez mayores, no solo en los niveles de educación formal de la población, sino en términos de una educación para toda la vida. La educación superior deberá realizar un esfuerzo para lograr una formación que combine todas las carreras y elementos de índole humanista, científica y técnica. Es necesario construir un sistema de educación superior abierto, en el que todas las instituciones y sectores participantes aporten y reciban elementos valiosos para un buen funcionamiento conjuntamente.

Ahora bien, junto con las características que especifican a la educación como un proceso que dura toda la vida y que concibe posible formar conocimientos y valores, debe existir la ineludible apertura para la actualización de los saberes y la posibilidad de instituir una escala de valores a la cual ellos respondan. Lo anterior es importante porque, entre las numerosas crisis que vivimos, la de los valores no es la menor (Castro, Rodríguez, Pujals, Durán y Díaz, 2012).

Cabe señalar que los estudiantes que se forman en las universidades deben caracterizarse por su capacidad en el desempeño laboral, científico, técnico, con consistentes principios éticos, políticos e ideológicos, todo ello acorde a los requerimientos de la profesión (López, 2013). En otras palabras, se exige la contextualización y personalización consecuente en cuanto a conocimientos y valores para el desarrollo del proceso de enseñanza-aprendizaje y, por tanto, la formación como profesionales con los requerimientos que demanda la sociedad actual. Integrar los valores al aprendizaje de modo intencional y consciente significa no solo pensar en el contenido como conocimientos y habilidades, sino en la relación que ellos poseen con los valores. Para resaltar los retos que se propone la educación superior sobre la educación en valores humanos es indiscutible la necesidad de apoyar el desarrollo de este proceso para su ocurrencia en los estudiantes (Socarrás, 2012). 


\section{Revista Iberoamericana de las Ciencias Sociales y Humanísticas}

Si bien la familia es la primera instancia en donde se vinculan los roles que promueven los valores que subyacen a los derechos humanos fundamentales, la libertad, la igualdad, el respeto, la solidaridad y el diálogo como forma de resolución de conflictos (Cortina, 1997), y que tienen que ver con la naturaleza de las personas, con las diferencias entre hombres y mujeres, se puede certificar que la educación superior es una importante vía para engendrar profesionales cuya actuación demuestre que han recibido una formación integral. La educación en valores en el nivel de educación superior es una actividad a cargo de los docentes, que debe realizarse en el contexto de la formación profesional del estudiante. Así pues, se encuentra y efectúa a través de las actividades curriculares y extracurriculares que corresponden con el proceso cognitivo (López, 2011).

Lo cierto es que los cambios tecnológicos, económicos, políticos y sociales desarrollados en las últimas décadas han concedido la edificación de una nueva estructura social, y con ella otras dinámicas relacionales que marcan un nuevo ciclo en la vida de las personas. En esa línea, Ceballos y Rodríguez (2017) plantean que en la estructura de parejas de doble ingreso la mujer es quien experimenta un mayor estrés, debido a que se encuentra más atada a una doble jornada, ya que al cumplir con su jornada laboral tiene la obligación de llegar a casa y cumplir con otra, aunque esta no remunerada: la de las tareas del hogar.

Aún más, Díaz, Dema, y Finkel (2015) hicieron una comparativa de las actividades remuneradas entre mujeres y hombres y observaron que las mujeres obtienen un menor ingreso que su pareja. Para medir la desigualdad utilizaron el planteamiento de Sorensen y McLanahan (1987) quienes proponen un indicador de dependencia económica de intrapareja: una persona es dependiente económicamente cuando sus ingresos son inferiores, en un $15 \%$ o más, a los de su pareja, lo cual es suficiente para decir que existe una relación desigual. Díaz et al. (2015) también plantean que los varones, conforme van cumpliendo años, van aportando más ingreso al hogar; mientras que, paralelamente, hay una disminución en las mujeres que aportan más o igual que sus compañeros. Por consiguiente, si ellas van cumpliendo más edad, van aportando o ganando menos que ellos. En suma, a mayor edad por parte de la mujer mayor dependencia económica hacia con su pareja. 


\section{Revista Iberoamericana de las Ciencias Sociales y Humanísticas}

Por su parte Castillo (2008) disecciona la teoría de dominancia social, enfoque que favorece la discriminación de género. Según esta teoría, los hombres presentan un mayor nivel de dominancia social en comparación con las mujeres debido a razones evolutivas y a estrategias de reproducción. Además, postula que las sociedades minimizan el conflicto grupal generando ideologías que promueven la superioridad de un grupo sobre los otros.

Las ideologías que promueven o mantienen la existencia de jerarquías y, por tanto, la desigualdad grupal, se pueden convertir en herramientas que legitiman la discriminación, y convienen ser considerablemente aceptadas por la sociedad para que surtan efecto, llegando a presentarse prácticamente como verdades obvias.

Las clasificaciones de las jerarquías sociales van desde las basadas en la edad hasta las fundamentadas en el género, sin dejar de lado aquellas construidas arbitrariamente: raza, orientación sexual, la religión, entre otras. (Castillo, 2008).

Campos y Rodríguez (2015) señalan a los estereotipos como otro factor de relevancia, y sumamente influyente en esta teoría de dominación social, ya que a través de ellos se les ha enseñado tanto a hombres como mujeres a cómo regir sus conductas, formas de vestir, actitudes; sin duda, a través de estos estereotipos el hombre se ha visto más beneficiado y valorado que la mujer, si bien, actualmente, existe una pequeña población en donde las mujeres son las que asumen este rol de mayor poder en la pareja y esto se debe a que tienen un nivel de escolaridad más alto que la pareja, mayor nivel adquisitivo económico.

Dema (2006) describe que aunque la mujer haya logrado un lugar en el ámbito laboral, no le favorece, ya que no existe una igualdad de roles en el hogar, por lo que existe una desventaja respecto a la carga de trabajo. En efecto, en comparación con las amas de casa tradicionales, las mujeres que laboran pueden realizarse como profesionistas, trabajadoras y lograr obtener una cierta independencia económica; sin embargo, el trabajo remunerado no asegura un equilibrio favorable entre la pareja.

\section{Objetivo}

El objetivo de la presente investigación consistió en identificar valores en la educación universitaria que reconocieran la equidad de género en parejas de doble ingreso que laboraron en un departamento gubernamental del estado de Zacatecas en el año 2019. 


\section{Método}

Para comprobar el objetivo de esta investigación, se elaboró un instrumento con 27 preguntas, conjuntamente con los datos generales. El instrumento fue validado por expertos en el entorno de la aplicación. La escala de respuestas fue de tipo Likert, con cinco opciones: desde "Nunca" hasta "Siempre". Las preguntas enunciadas a negativo se recodificaron para el análisis. Se realizó una prueba piloto (mayo de 2019) con 35 preguntas, la cual fue aplicada a 21 personas, para probar la comprensión, el orden y las dificultades o resistencias al responder a las preguntas y su respectiva validación con el alfa de Cronbach (1951). De lo anterior se obtuvieron que cinco preguntas resultaron redundantes, por ello la estructura fue rediseñada para una mejor comprensión. Por las particularidades del presente estudio y analizando los tipos de enfoques, la investigación se tornó cuantitativa, de tipo transaccional o transversal, al tratarse de una investigación que compila datos en un momento único. En este estudio se recolectó información en un momento determinado para obtener los resultados pertinentes. Para efectos del presente proyecto, el nivel de la investigación fue relacional, ya que se encontraron relaciones entre variables de equidad de género en parejas de doble ingreso, lo cual sirvió para determinar la relación que hubo entre ellas y las discrepancias mediante la prueba de ji al cuadrado de Pearson (Hernández, Fernández y Baptista, 2010). La población de estudio fueron los trabajadores de un departamento gubernamental del estado de Zacatecas ubicado en Boulevard Héroes de Chapultepec 1902, Ciudad Administrativa, a partir de la cual determinamos una muestra por conveniencia de 65 trabajadores, los cuales cumplían con los requisitos de estar en un rango de edad de entre 20 y 60 años, además de ser parte de una pareja de doble ingreso (33 mujeres y 32 hombres).

\section{Resultados}

Al realizar un análisis de las frecuencias obtenidas de las variables de naturaleza ordinal, en escala tipo Likert, de acuerdo con la opinión de los encuestados, se encontró que los datos se pueden agrupar de la siguiente manera.

En cuanto a la equidad de género, según se observa en la tabla 2, la mayor parte de los encuestados afirmó que el hombre participa en actividades domésticas; algunas veces no apoyan a su pareja, pero no sienten una carga al realizarlas ni al dejar de hacerlas. 
Revista lberoamericana

de las Ciencias Sociales y Humanísticas

Figura 1. Equidad de género en el hogar

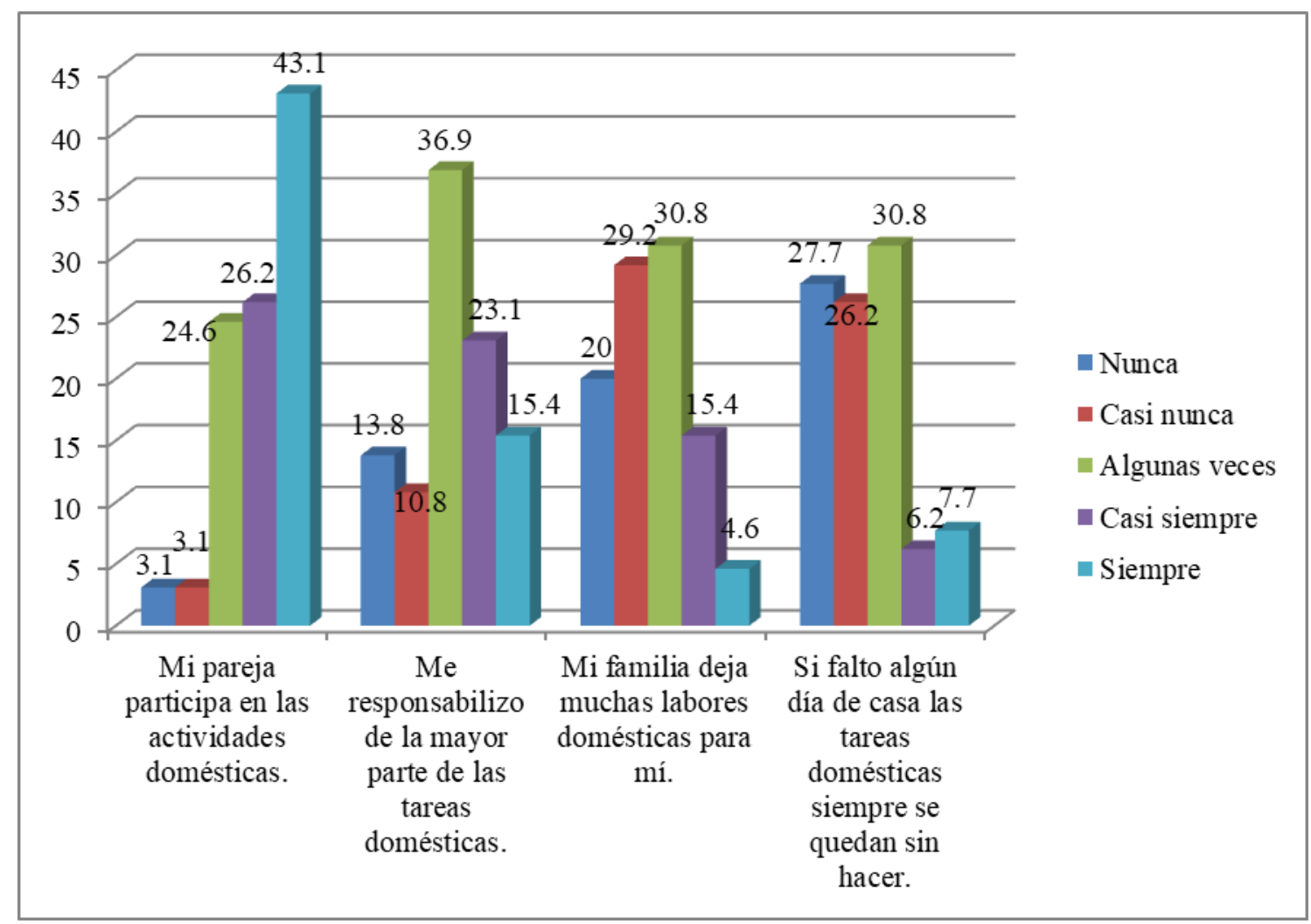

Nota: el gráfico muestra los porcentajes referentes a las labores del hogar.

Fuente: elaboración propia.

Respecto a la equidad y economía familiar, como se muestra en la figura 2, un gran porcentaje declinó sentir preocupación por necesitar ayuda con los gastos del hogar. Asimismo, la mayoría algunas veces recurre a su pareja cuando necesitan de apoyo económico. Es importante recalcar que el estudio denota que las parejas se sienten siempre valoradas al aportar económicamente en los gastos del hogar. 
Revista lberoamericana

de las Ciencias Sociales y Humanísticas

Figura 2. Equidad y economía familiar

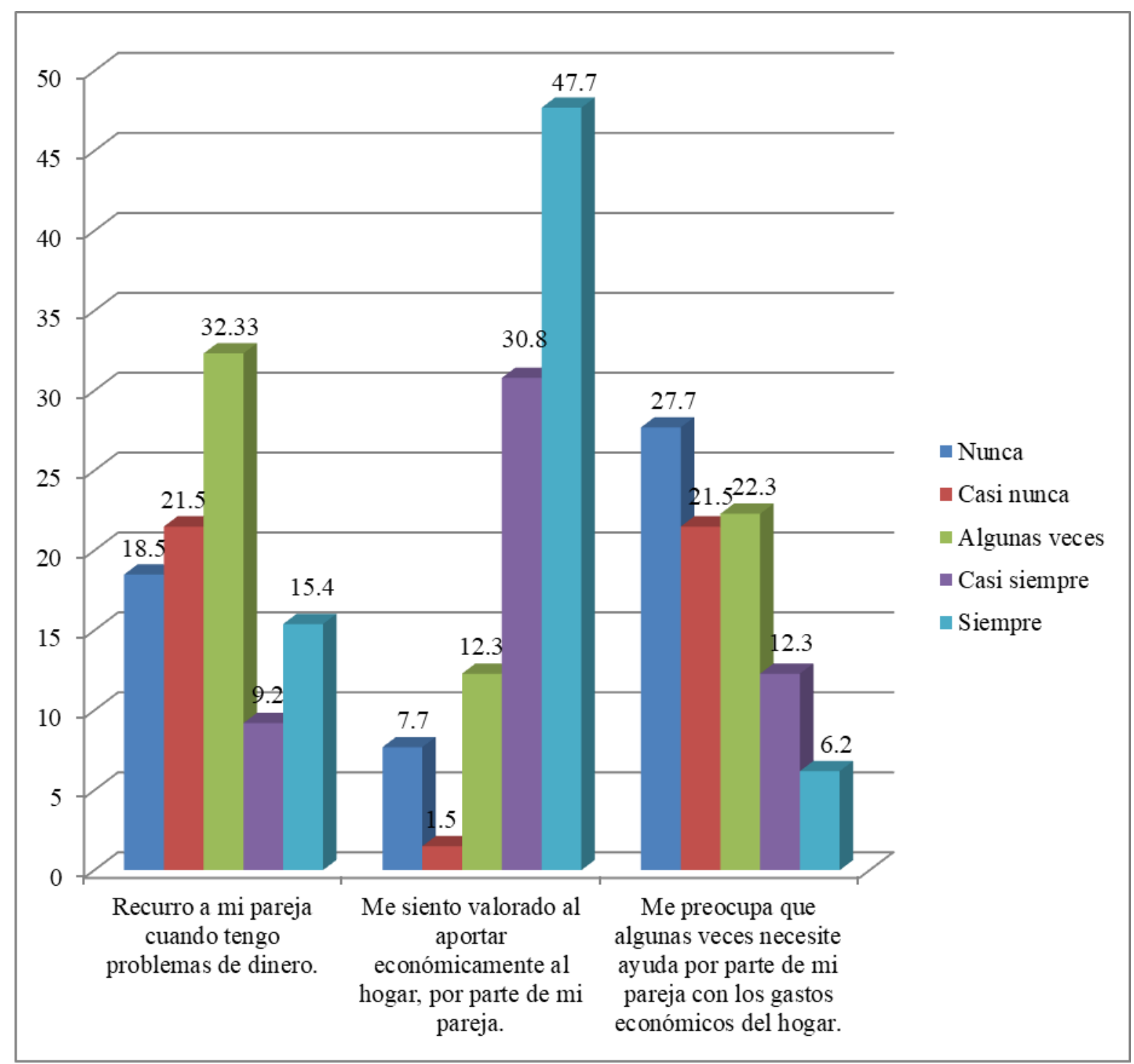

Nota: el gráfico muestra los porcentajes en relación con la equidad y economía familiar.

Fuente: Elaboración propia

Mientras que, en cuanto a la equidad de género y conflictos de pareja, de acuerdo con la figura 3, los participantes, en su mayoría, siempre sintieron apoyo de la pareja en sus ámbitos laborales, además de que nunca consideraron que sus derechos fueran vulnerados. 
Revista lberoamericana

de las Ciencias Sociales y Humanísticas

Figura 3. Equidad y conflictos de pareja

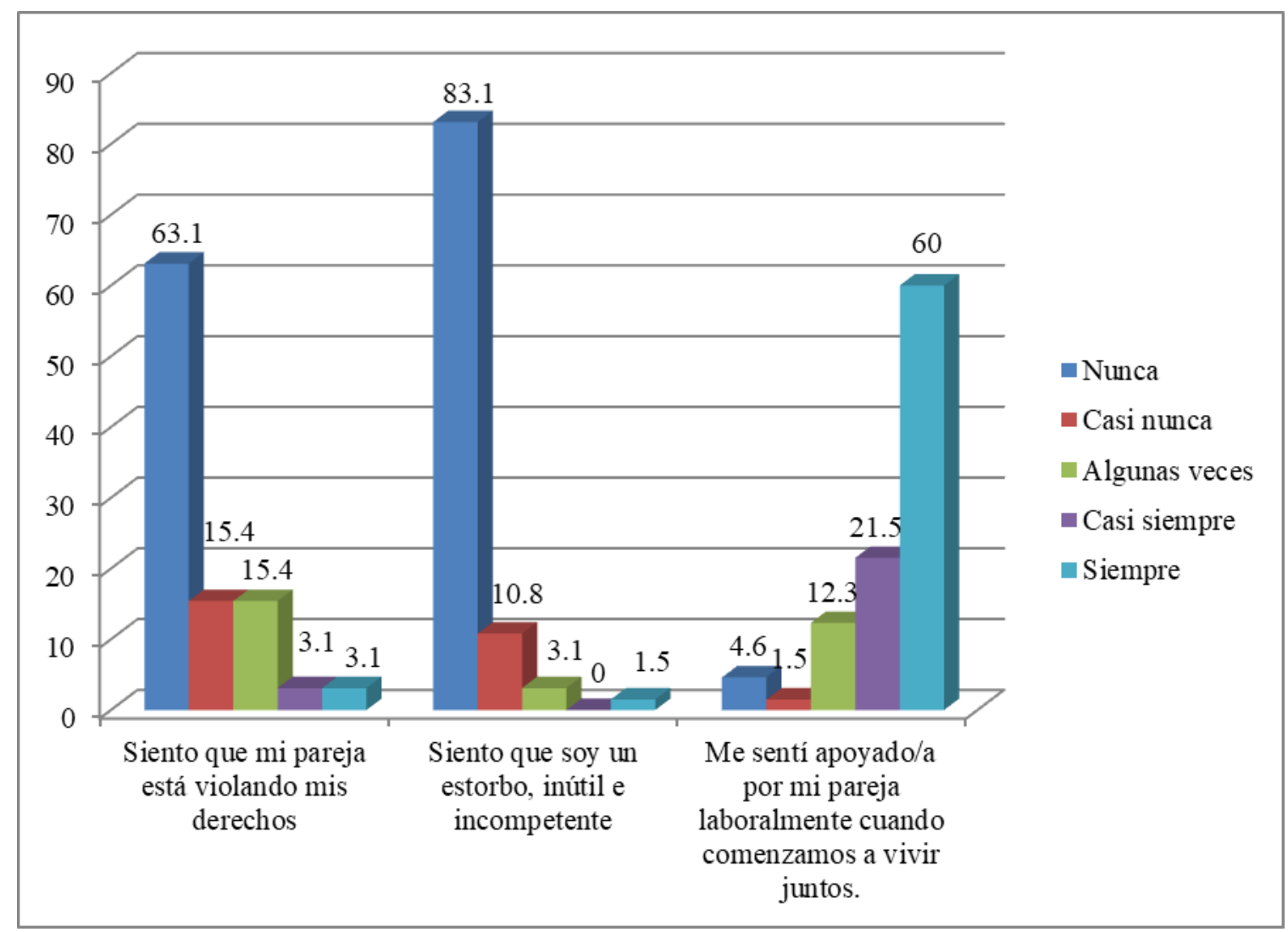

Nota: el gráfico muestra los porcentajes en relación con la equidad y conflictos de pareja.

Fuente: Elaboración propia

En la tabla 1, por su parte, se muestran las frecuencias obtenidas de los ítems más importantes y se destacan aquellas que, según el estadístico ji al cuadrado, resultaron significativas a $5 \%$ (significancia asintótica bilateral menor o igual a 0.05 ). 
Revista lberoamericana

de las Ciencias Sociales y

Humanísticas

Tabla 1. Frecuencias obtenidas

\begin{tabular}{|l|l|l|l|l|l|}
\hline & Nunca & $\begin{array}{l}\text { Casi } \\
\text { nunca }\end{array}$ & $\begin{array}{l}\text { Algunas } \\
\text { veces }\end{array}$ & $\begin{array}{l}\text { Casi } \\
\text { siempre }\end{array}$ & Siempre \\
\hline $\begin{array}{l}\text { ¿Con qué frecuencia influye su } \\
\text { pareja en su bienestar? (Por } \\
\text { ejemplo: disgustos, problemas de } \\
\text { salud, intranquilidad, etc.). }\end{array}$ & 15.4 & 32.3 & 24.6 & 15.4 & 10.8 \\
\hline $\begin{array}{l}\text { Mi vida laboral interfiere con mis } \\
\text { actividades sociales, como visitar } \\
\text { a mis familiares. }\end{array}$ & 30.8 & 26.2 & 32.3 & 7.7 & 3.1 \\
\hline $\begin{array}{l}\text { Mi vida laboral interfiere con mis } \\
\text { actividades sociales, como visitar } \\
\text { a mis amigos. }\end{array}$ & 33.8 & 26.2 & 24.6 & 7.7 & 6.2 \\
\hline $\begin{array}{l}\text { Tengo suficiente energía para mis } \\
\text { actividades cotidianas. }\end{array}$ & 3.1 & 7.7 & 15.4 & 38.5 & 35.4 \\
\hline $\begin{array}{l}\text { Siento que soy un estorbo, inútil e } \\
\text { incompetente. }\end{array}$ & 83.1 & 10.8 & 3.1 & 0 & 1.5 \\
\hline $\begin{array}{l}\text { Me he sentido preocupado/a por } \\
\text { mis finanzas. }\end{array}$ & 4.6 & 10.8 & 55.4 & 13.8 & 13.8 \\
\hline $\begin{array}{l}\text { Siento que mi pareja está } \\
\text { violando mis derechos. }\end{array}$ & 63.1 & 15.4 & 15.4 & 3.1 & 3.1 \\
\hline $\begin{array}{l}\text { Me siento socialmente } \\
\text { competente. }\end{array}$ & 7.7 & 0 & 6.2 & 30.8 & 55.4 \\
\hline $\begin{array}{l}\text { Mi horario de trabajo a menudo } \\
\text { choca con mi vida familiar. }\end{array}$ & 27.7 & 21.5 & 36.9 & 7.7 & 6.2 \\
\hline $\begin{array}{l}\text { Estoy satisfecho/a con el tiempo } \\
\text { que le dedico a mi familia. }\end{array}$ & 7.7 & 7.7 & 24.6 & 30.8 & 27.7 \\
\hline $\begin{array}{l}\text { Mis obligaciones del trabajo y } \\
\text { mis obligaciones en la familia son } \\
\text { compatibles. }\end{array}$ & 12.3 & 6.2 & 23.1 & 32.3 & 23.1 \\
\hline $\begin{array}{l}\text { Después del trabajo llego a casa } \\
\text { demasiado cansado/a para hacer }\end{array}$ & 15.4 & 18.5 & 46.2 & 10.8 & 9.2 \\
\hline
\end{tabular}


Revista Iberoamericana

de las Ciencias Sociales y

Humanísticas

ISSN: 2395 - 7972

\begin{tabular}{|l|l|l|l|l|l|}
\hline lo que me gusta. & & & & & \\
\hline $\begin{array}{l}\text { Han surgido problemas con mi } \\
\text { pareja. }\end{array}$ & 15.4 & 46.2 & 32.3 & 6.2 & 0 \\
\hline $\begin{array}{l}\text { Mi pareja cubre con sus } \\
\text { necesidades económicas. }\end{array}$ & 15.4 & 18.5 & 27.7 & 16.9 & 20.0 \\
\hline $\begin{array}{l}\text { Mi pareja participa en las } \\
\text { actividades domésticas. }\end{array}$ & 3.1 & 3.1 & 24.6 & 26.2 & 43.1 \\
\hline $\begin{array}{l}\text { Mi pareja me ha agredido } \\
\text { físicamente. }\end{array}$ & 83.1 & 7.7 & 7.7 & 0 & 1.5 \\
\hline $\begin{array}{l}\text { Me preocupa que algunas veces } \\
\text { necesite ayuda por parte de mi } \\
\text { pareja con los gastos económicos } \\
\text { del hogar. }\end{array}$ & 27.7 & 21.5 & 22.3 & 12.3 & 6.2 \\
\hline $\begin{array}{l}\text { Recurro a mi pareja cuando tengo } \\
\text { problemas de dinero. }\end{array}$ & 18.5 & 21.5 & 32.33 & 9.2 & 15.4 \\
\hline $\begin{array}{l}\text { Puedo hablar con mi pareja sobre } \\
\text { decisiones importantes. }\end{array}$ & 3.1 & 4.6 & 9.2 & 20.0 & 63.1 \\
\hline $\begin{array}{l}\text { Si necesito arreglar algo en casa, } \\
\text { cuento con mi pareja para que me } \\
\text { ayude. }\end{array}$ & 20.0 & 29.2 & 30.8 & 15.4 & 4.6 \\
\hline $\begin{array}{l}\text { Me siento valorado/a al aportar } \\
\text { económicamente al hogar por } \\
\text { parte de mi pareja. }\end{array}$ & 7.7 & 1.5 & 12.3 & 30.8 & 47.7 \\
\hline $\begin{array}{l}\text { Me sentí apoyado/a por mi pareja } \\
\text { laboralmente cuando } \\
\text { comenzamos a vivir juntos. }\end{array}$ & 4.6 & 1.5 & 12.3 & 21.5 & 60.0 \\
\hline $\begin{array}{l}\text { Si falto algún día de casa las } \\
\text { tareas domésticas siempre se } \\
\text { quedan sin hacer. }\end{array}$ & 27.7 & 26.2 & 30.8 & 6.2 & 7.7 \\
\hline $\begin{array}{l}\text { Mi familia deja muchas labores } \\
\text { domésticas para mí. }\end{array}$ & 20.0 & 16.9 & 60.0 \\
\hline
\end{tabular}


Revista Iberoamericana

de las Ciencias Sociales y

Humanísticas

ISSN: $2395-7972$

\begin{tabular}{|l|l|l|l|l|l|}
\hline $\begin{array}{l}\text { Me responsabilizo de la mayor } \\
\text { parte de las tareas domésticas. }\end{array}$ & 13.8 & 10.8 & 36.9 & 23.1 & 15.4 \\
\hline $\begin{array}{l}\text { Mi familia me agobia con cosas } \\
\text { que deberían ser capaces de hacer } \\
\text { por sí mismos. }\end{array}$ & 29.2 & 23.1 & 35.4 & 10.8 & 1.5 \\
\hline $\begin{array}{l}\text { A menudo necesito estar en el } \\
\text { trabajo y en casa a la vez. }\end{array}$ & 26.2 & 20.0 & 38.5 & 13.8 & 1.5 \\
\hline
\end{tabular}

Fuente: Elaboración propia

Al analizar las frecuencias obtenidas en la tabla 1, de acuerdo con las respuestas de las cuestiones planteadas en el instrumento de manera general, se encontró lo siguiente:

- $\quad$ Consideraron que siempre se sintieron socialmente competentes, valorados por la pareja al aportar económicamente, además de que esta le apoyó con las actividades del hogar, y mostraron confianza al hablar de decisiones importantes.

- Casi siempre percibieron tener suficientes energías para las actividades cotidianas, sentirse satisfechos por el tiempo dedicado a la familia, así como la compatibilidad entre ellos y el trabajo.

- Opinaron que algunas veces su trabajo interfirió con sus actividades sociales, su horario de trabajo coincidió con su vida familiar y manifestaron que llegaron a sentir cansancio para hacer lo que les gustaba, además de que estuvieron preocupados por las finanzas, a pesar de que su pareja cubría con las necesidades económicas y recurrieron a su ayuda cuando se presentaban problemas de esta índole; en relación con las actividades domésticas, consideraron que se quedaron sin hacer, o dejaron muchas actividades, así como revelaron que tuvieron que hacer estas actividades al mismo tiempo que el trabajo.

- $\quad$ Casi nunca tuvieron la percepción de que el trabajo influyó en su bienestar. Igualmente, casi nunca presentaron problemas de salud, disgustos o discusiones.

- $\quad$ Respondieron que su pareja nunca les agredió físicamente, nunca se sintieron un estorbo, inútil e incompetente, y que el trabajo interfirió en algunas actividades sociales como visitar amigos. 


\section{Discusión}

Las instituciones de educación superior poseen un papel importante en el proceso de adquisición de los modelos de conducta que aprenden las personas referentes a los patrones de relación entre hombres y mujeres. En la escuela, de una manera paralela al currículo formal, se despliega de forma incluida un currículo oculto, cimentado en un modelo de relación entre los géneros, donde el modelo de masculinidad absoluto, personalizado por los estereotipos y la diferencia, sigue estando presente (Santos, 2006). Si bien se ha evolucionado mucho en las últimas décadas en relación con la equidad, no obstante, en la "escuela de todos" sigue implementándose un sistema desigual para los géneros que continúa observándose natural en la sociedad en la que vivimos (Piussi, 1997).

Por ello es ineludible seguir adecuando y poniendo en práctica en las instituciones educativas medidas que originen la igualdad real y efectiva entre géneros. De acuerdo con Gallardo y Vázquez (2018), la educación escolar instituye un espacio óptimo para edificar un mundo cimentado en igualdad de género que garantice la equidad. Una educación cimentada en valores como la libertad, la igualdad, el respeto, la comunicación y la negociación como camino para la resolución de conflictos en parejas de doble ingreso, la cooperación que aprecie las diferencias, la diversidad cultural y sexual de las personas de nuestra sociedad, promueve la transformación de los esquemas adquiridos de forma rígida en la primera infancia en relación con los géneros. La integración de niños y adolescentes en grupos de iguales en el entorno universitario marca las características de interacción horizontal intra e intergéneros, lo cual es uno de los soportes de la socialización escolar en relación con los modelos entre los géneros (Povedano, Muñiz, Cuesta y Musitu, 2015).

\section{Conclusiones}

El objetivo de la presente investigación consistió en identificar los valores en la educación universitaria que reconocieran la equidad de género en parejas de doble ingreso. Es importante retomar que la educación para la igualdad de género se cimienta en los valores éticos como lo es la libertad, igualdad, respeto, solidaridad, diálogo entre otros, y tiene como objetivo que hombres y mujeres se desenvuelvan intrínsecamente en un modelo social igualitario. Por lo tanto, derivado de esto, en la investigación realizada se observó que se ha incrementado la aparición de las mujeres en los ámbitos laborales; así, a mayor nivel de escolaridad por parte de la mujer se tiene una mayor negociación con la pareja para 


\section{Revista Iberoamericana \\ de las Ciencias Sociales y Humanísticas}

ISSN: $2395-7972$

llegar a un mutuo acuerdo en la repartición de actividades domésticas y pasatiempos en comparación con las mujeres que tienen un nivel de escolaridad más bajo. Del mismo modo, los resultados del análisis de los datos determinan valores en las parejas de doble ingreso que laboraron en este departamento gubernamental: el reconocimiento social, la competitividad, la valoración al realizar actividades del hogar y la confianza de hablar de decisiones importantes, así como el dar apoyo a su pareja ante cualquier situación económica. Por otro lado, algunos encuestados percibieron que no se sintieron valorados, ya que se sintieron agredidos física y psicológicamente, lo que les ocasionó problemas de salud, disgustos o discusiones. Los resultados confirman que las parejas de doble ingreso atraen más conflicto en comparación con las parejas "normales", ya que en la forma tradicional la mujer solamente se hace cargo de las labores domésticas y el hombre funge como proveedor; al entrar a la nueva estructura, las "parejas de doble ingreso" toman roles distintos a los ya establecidos por la sociedad: la mujer también realiza trabajo remunerado y el hombre apoya en las labores domésticas. Los matrimonios de doble ingreso (matrimonios modernos) se enfrentan a mayores problemas cotidianos en el trabajo, hogar y en la crianza de los hijos, por lo cual se ve afectado el modelo social igualitario, en el que los hombres y mujeres se desenvuelvan básicamente en un modelo social igualitario.

Es indispensable seguir apostando por procesos de intervención social respecto al género en las escuelas y por los programas de educación para la igualdad de género porque, como ya se ha subrayado anteriormente, son las vías para avanzar hacia un modelo social más igualitario. Igualmente, es fundamental tener en cuenta la difusión de las experiencias educativas valiosas en la implementación de los programas de coeducación en algunas escuelas en la medida en que puedan servir de gran ayuda para otras que todavía no han iniciado sus programas en coeducación o están en proceso de inicio. En este sentido, es sustancial contar con indicadores que ayuden a determinar el valor de una buena práctica educativa en los programas de educación en igualdad. 


\section{Futuras líneas de investigación}

Esta investigación ha abierto algunas líneas de investigación que se pueden desarrollar en un futuro inmediato, entre las cuales podemos mencionar las siguientes:

- La prevención de la violencia de género en parejas de doble ingreso, es necesario explorar contenidos temáticos en la educación con el fin de excluir los mensajes sexistas, además de diseñar acciones que divulguen las secuelas de los diferentes tipos de conductas que provocan trastornos en la vida de las mujeres. Asimismo, favorecer a la investigación para que apoye los problemas de salud con perspectiva de género; incluir en los programas académicos de las universidades cómo abordar el tema de la violencia, su frecuencia, manifestaciones, diagnóstico y tratamiento.

- La igualdad entre mujeres y hombres en el ámbito educativo universitario, la exploración de los programas tiene como objetivo identificar la presencia de gestiones encaminadas a la formación de docentes en materia de género; la inserción de contenidos en la educación y estrategias enfocadas a combatir la discriminación y promover la igualdad en la educación de hombres y mujeres.

- El trabajo doméstico y los valores en las parejas de doble ingreso, se ha observado que las mujeres se encuentran más enfocadas a la entrega del trabajo doméstico y bienestar de las personas del hogar. Las mujeres padecen una mayor desigualdad en el trabajo no remunerado dentro de los hogares.

Finalmente, hay que destacar la creación de políticas públicas para la igualdad de género. Los gobiernos estatales y la sociedad están obligados a desarrollar la reeducación de hombres y mujeres, por tanto, es elemental la contribución de las instituciones facultadas en la elaboración de políticas públicas en programas de sensibilización y formación que edifiquen una nueva visión de la masculinidad y feminidad en colaboración con la sociedad civil, de modo que conjuntamente formulen programas que fortalezcan una cultura de respeto a los derechos humanos. 
Revista Iberoamericana

de las Ciencias Sociales y

Humanísticas

ISSN: 2395 - 7972

\section{Referencias}

Campos, R. Lilia, \& Rodríguez-Shadow, María J. (2015). Toma de decisiones en las relaciones de pareja de las mujeres con poder adquisitivo elevado. Investigación y Ciencia, 23(66),56-63.[fecha de Consulta 10 de Diciembre de 2020]. ISSN: 16654412. Disponible en: https://www.redalyc.org/articulo.oa? $\mathrm{id}=674 / 67446014008$

Castillo, M. R. (2008). Aportaciones de la Teoría de la Dominancia Social al análisis de la discriminación de género. Iniciación a La Investigación, (3). 1-6. Recuperado a partir de https://revistaselectronicas.ujaen.es/index.php/ininv/article/view/200

Castro, B. M., Espinosa Rodríguez, R., Pujals Victoria, N., Durán García, F., \& Díaz Cruz, L. (2012). Lo social en el currículo de formación de profesionales de la salud. Educación Médica Superior, 26(3), 1-16. Recuperado de http://www.ems.sld.cu/index.php/ems/article/view/53

Ceballos, E. y Rodríguez, J. (2017). Uso del tiempo y conflicto familiar. International Journal of Developmental and Educational Psychology, 6(1), 77-84.

Cortina, A. (1997). Ciudadanos del mundo. Hacia una teoría de la ciudadanía. Madrid, Alianza.

Cronbach, L. J. (1951). Coefficient alpha and the internal structure of tests. Psychometrika, 16, 297-334.

Dema, S. (2006). Una pareja de salarios: El dinero y las relaciones de poder en las parejas de doble ingreso. Madrid, España: CIS, Siglo XXI.

Díaz, C., Dema, S. y Finkel, L. (2015). Desigualdades de género en la distribución de los recursos económicos en las parejas. Papers: Revista de Sociologia, 100(1), 53-73.

Gallardo, J. A. y Vázquez, P. (2018). Equidad e igualdad de género en educación. Ponencia presentada en el IV Congreso Virtual Internacional sobre Innovación Pedagógica y Praxis Educativa. Sevilla, del 20 al 22 de marzo de 2018.

Hernández, R., Fernández, C. y Baptista, M. (2010) Metodología de la investigación (5. ${ }^{a}$ ed.). Ciudad de México, México: McGraw-Hill.

López, M. (2011). La educación y derechos humanos: Una asignatura orientada a favorecer la convivencia. Barcelona, España: Universidad de Barcelona.

López, R. (2013). Ética profesional en la formación universitaria. Perfiles Educativos, 35(142), 15-24. 
Piussi, A. M. (1997). Enseñar ciencia: autoridad femenina y relaciones en la educación. Icaria Editorial.

Povedano, A., Muñiz, M., Cuesta, P. y Musitu, G. (2015). Educación para la igualdad de género. Un modelo de evaluación. Madrid, España: Centro Reina Sofía sobre Adolescencia y Juventud.

Santos, M. A. (2006). La escuela que aprende. Retos, dificultades y esperanzas. Madrid, España: Ediciones Morata.

Socarrás, S. (2011). Manual del profesor guía para el perfeccionamiento del trabajo educativo. Humanidades Médicas, 11(2), 345-359.

Sorensen, A. and Mclanahan, S. (1987). Married Women's Economic Dependency: 19401980. American Journal of Sociology, 93(3), 659-687. 
Revista Iberoamericana

de las Ciencias Sociales y

Humanísticas

ISSN: 2395 - 7972

\begin{tabular}{|c|c|}
\hline Rol de Contribución & Autor (es) \\
\hline Conceptualización & Flor de María García Martínez \\
\hline Metodología & $\begin{array}{l}\text { Flor de María García Martínez (Igual) y Víctor Hugo } \\
\text { Bañuelos García (Igual) }\end{array}$ \\
\hline Software & Víctor Hugo Bañuelos García \\
\hline Validación & $\begin{array}{l}\text { Víctor Hugo Bañuelos García (principal) y Flor de María } \\
\text { García Martínez (apoyo) }\end{array}$ \\
\hline Análisis Formal & Víctor Hugo Bañuelos García \\
\hline Investigación & $\begin{array}{l}\text { Flor de María García Martínez (principal) y Blanca Isabel } \\
\text { Llamas Félix (apoyo) }\end{array}$ \\
\hline Recursos & $\begin{array}{l}\text { Víctor Hugo Bañuelos García (Igual) y Blanca Isabel Llamas } \\
\text { Félix (Igual) }\end{array}$ \\
\hline Curación de datos & $\begin{array}{l}\text { Víctor Hugo Bañuelos García (principal) y Flor de María } \\
\text { García Martínez (apoyo) }\end{array}$ \\
\hline $\begin{array}{l}\text { Escritura - Preparación del } \\
\text { borrador original }\end{array}$ & $\begin{array}{l}\text { Flor de María García Martínez (principal) y Blanca Isabel } \\
\text { Llamas Félix (apoyo) }\end{array}$ \\
\hline $\begin{array}{l}\text { Escritura - Revisión y } \\
\text { edición }\end{array}$ & Flor de María García Martínez \\
\hline Visualización & $\begin{array}{l}\text { Flor de María García Martínez (principal) y Víctor Hugo } \\
\text { Bañuelos García (apoyo) }\end{array}$ \\
\hline Supervisión & Flor de María García Martínez \\
\hline $\begin{array}{l}\text { Administración de } \\
\text { Proyectos }\end{array}$ & Flor de María García Martínez \\
\hline Adquisición de fondos & $\begin{array}{l}\text { Flor de María García Martínez (igual) y Blanca Isabel Llamas } \\
\text { Félix (igual) }\end{array}$ \\
\hline
\end{tabular}

